\title{
FABRICATION AND CHARACTERIZATION OF MEMS BASED WAFER-SCALE PALLADIUM-SILVER ALLOY MEMBRANES FOR HYDROGEN SEPARATION AND HYDROGENATION/DEHYDROGENATION REACTIONS
}

\author{
H.D.Tong ${ }^{\text {a }}$, F.C.Gielens ${ }^{\text {b }}$, J.W.Berenschot ${ }^{\text {a }}$, M.J.De Boer ${ }^{\text {a }}$, J.G.E.Gardeniers ${ }^{\text {c }}$, W.Nijdam ${ }^{\text {a, }}$ C.J.M. van Rijn ${ }^{\text {a }}$, \\ M.C.Elwenspoek ${ }^{\mathrm{a}}$ \\ ${ }^{a}$ Transducer Science Technology Group, MESA+ research institute, University of Twente, The Netherlands \\ ${ }^{b}$ Department of Chemical Engineering and Chemistry, Eindhoven University of Technology, The Netherlands \\ ${ }^{c}$ Micronit Microfluidics B.V., The Netherlands \\ E-mail: T.Hien@el.utwente.nl; Tel: X-31-53-4892805
}

\begin{abstract}
In this paper, a MEMS based wafer-scale palladium - silver alloy membrane (MWSPdAgM) is presented. This membrane has the potential to be used for hydrogen purification and other applications [1]. A palladium-silver alloy layer ( $\mathrm{Pd}-\mathrm{Ag})$ was prepared by co-sputtering. The thin Pd-Ag alloy has high hydrogen selectivity, high permeation rate as well as high mechanical and chemical stability. Typical flow rates of $0.5 \mathrm{~mol} \mathrm{H}_{2} / \mathrm{m}^{2}$.s have been measured with a minimal selectivity of 550 for $\mathrm{H}_{2} / \mathrm{N}_{2}$. Anodic bonding of thick glass to silicon was used to package the membrane and create a very robust module. The membrane has high mechanical strength and can withstand pressures up to 4 bars at room temperature. The presented fabrication method allows the development of a module for industrial applications that consists of a stack with a large number of glass/membrane plates.
\end{abstract}

\section{INTRODUCTION}

The increased demand for hydrogen in recent years in many sectors such as petrochemical and semi-conductor processing and fuel cell applications has led to a revival of interest in methods for separation and purification of hydrogen from gas mixtures [2]. Palladium (Pd)-based membranes have been the focus of many studies, due largely to their unmatched potential as hydrogen-selective membranes for purification. A comprehensive review of Pd-based membranes and their applications has been recently been provided by R.Dittmeyer et al. [1] and by Shu et al. [3].

However, conventional technology is limited by the high cost of palladium combined with fabrication methods resulting in thick palladium films. For instance, palladium/silver metal multitubular assemblies having a membrane thickness of
$50 \mu \mathrm{m}$ or more have been used for many years for laboratory purification of hydrogen. The wall thickness of these tubes incorporated in such assemblies reduces the hydrogen flux and inhibits their application for larger-scale chemical production, apart from the investment cost of the precious metal.

Microtechnology is able to produce very thin Pd membranes, therewith dramatically increasing the flux efficiency of the membrane, and possibly decreasing the cost of the technology. So far, only chip-diced Pd membranes have been reported, e.g. at MEMS' 99 [4]. In this paper, the process development of a wafer-scale separation membrane is presented. The membrane performance was also characterized with respect to mechanical strength, hydrogen flux, and selectivity.

\section{FABRICATION}

Palladium Silver Membranes on Silicon Frame

A cross-section of the PdAg membrane is shown in figure 1. It consists of a silicon wafer and two glass wafers.

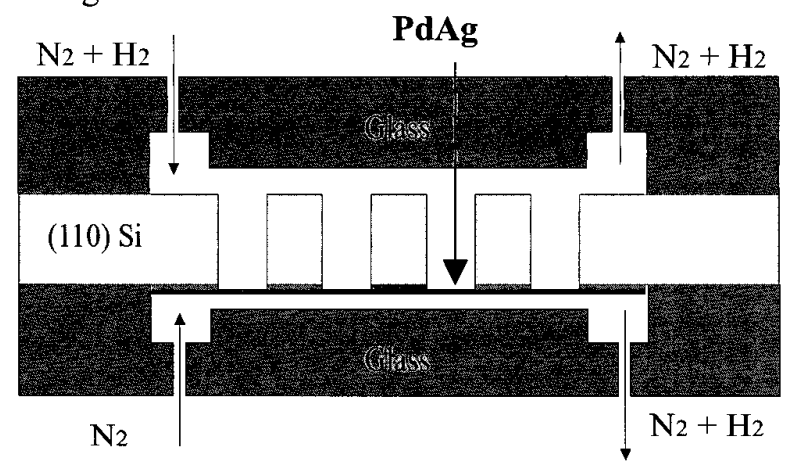

Figure 1: Cross-section of the separation membrane 
The process steps of the membrane are shown in figure 2. A 3 inch, (110), double side polished silicon substrate is coated with $1 \mu \mathrm{m}$ of wetthermally oxidized $\mathrm{SiO}_{2}$. The $\mathrm{SiO}_{2}$ coating serves as a protective layer during subsequent etching steps. It should be mentioned that, for the fabrication of highaspect-ratio features by anisotropic etching of (110) silicon, precise alignment of the features to the (111) planes is of critical importance and was described in detail in $[5,6]$. First of all, fan-shaped structures are imprinted on the silicon wafer by standard photolithography, followed by removal of $\mathrm{SiO}_{2}$ in a buffered oxide etch (BHF 1:7). Afterwards, the fanshaped structures are etched for a short time in $\mathrm{KOH}$ to indicate the exact $\langle 110\rangle$ directions [6]. Then, narrow slits of 25 by $1250 \mu \mathrm{m}$ are aligned to $<110>$ directions and patterned using the steps mentioned above. The wafer is immersed in $25 \% \mathrm{KOH}$ solution at $75^{\circ} \mathrm{C}$ to etch the silicon until ca. $50 \mu \mathrm{m}$ is left. A similar pattern may also be obtained with Reactive Ion Etching [5]. However, with that method only one wafer at a time can be processed while several can be etched simultaneously with $\mathrm{KOH}$. An alloy layer of $\mathrm{Pd} 77 \%-\mathrm{Ag} 23 \%$ w with a thickness of $1 \mu \mathrm{m}$ is deposited by co-sputtering [7] through a shadow mask on the bottom side of the silicon wafer, using titanium (Ti) as an adhesion layer. In our study, PdAg is used as separation element because it has shown higher resistance to hydrogen embrittlement than pure Pd [8]. Next, $\mathrm{KOH}$ is used to etch silicon in the trenches until the $\mathrm{SiO}_{2}$ layer is reached. Finally, this oxide layer and the $\mathrm{Ti}$ are removed in BHF to reveal the back surface of the Pd-Ag membranes. The supported silicon structures and the membrane are shown in figure 3 and figure 4 .

\section{Flow Channels in Glass}

A powder blasting technique, which has been developed in our lab [9], was used to create a flow channel of $1 \mathrm{~mm}$ depth on each of two $5 \mathrm{~mm}$ thick glass wafers. The flow channel is then connected to the outer world by four previous drilled holes within the glass wafers. A picture of the flow channels is shown in figure 5 .

\section{Assembly of Wafer Module}

Finally, the silicon wafer is bonded between the two thick glass wafers by a four-electrode anodic

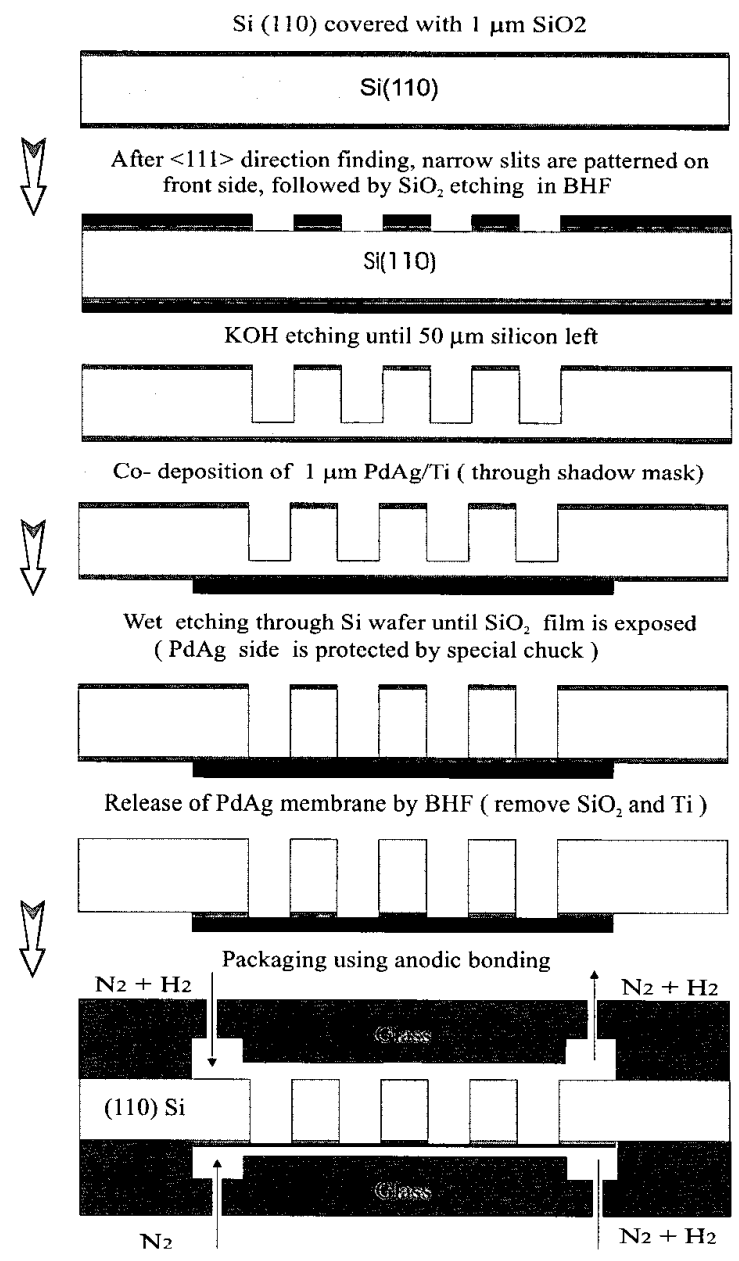

Figure 2: Fabrication process of the membrane

bonding technique (see figure 6). The process is performed in two steps, because adequate bonding requires that a positive electrical voltage be applied to the silicon and a negative voltage to the glass. This process results in a tight seal between each glass wafer and the silicon wafer.

A stainless steel membrane holder was developed and is presented in figure 7. In the design, graphite rings are used to make a gas-tight connection.

\section{RESULTS AND DISCUSSION}

Preliminary tests showed that the PdAg membranes do not break at a pressure difference of 4 bars between the two sides of the membrane. This is much higher than expected on the basis of literature data for bulk Pd[10]. 


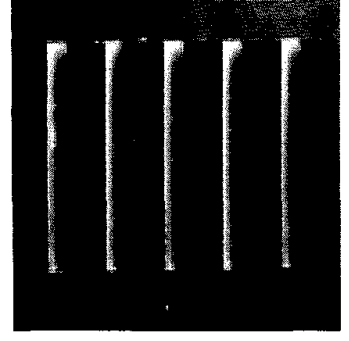

(a)

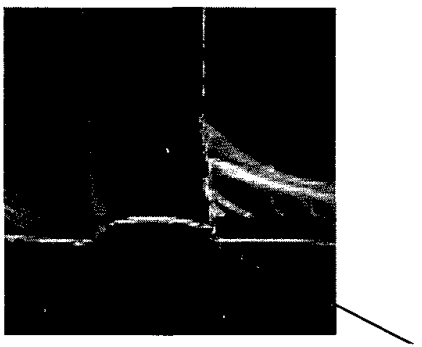

(b)

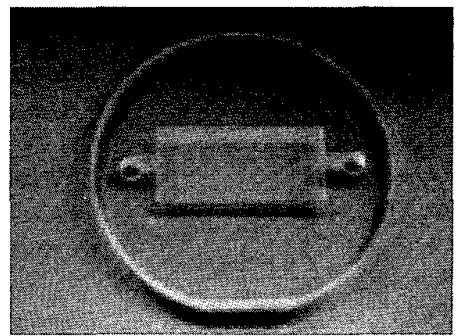

PdAg

Figure 3: SEM pictures of the support structures etched in (110) silicon wafer (a) and close up of cross-section of the PdAg membrane (b)

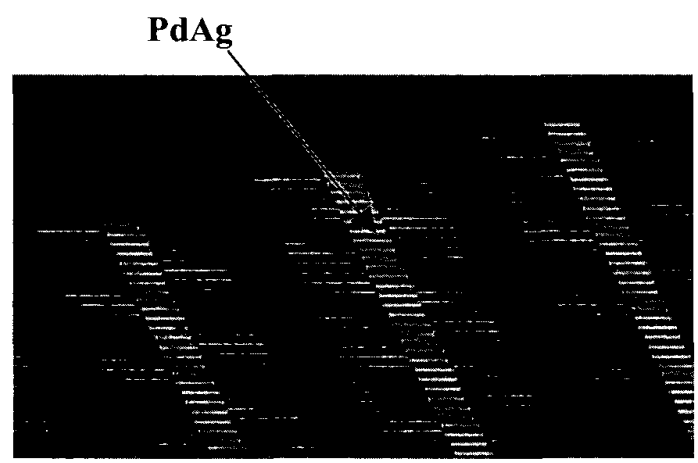

Figure 4: Top view of the PdAg membrane through $\mathrm{Si}$ supported structure. The white areas are free PdAg membranes. The black areas are (111) planes as seen through $\mathrm{Si}$ frame.

To determine hydrogen permeation and selectivity of the membranes, they were positioned in the stainless steel membrane holder and heated up to elevated temperatures. Next, the membrane permeability and selectivity for hydrogen were determined as a function of the hydrogen partial pressure $(0-0.3 \mathrm{bar})$, and temperature $\left(350-450^{\circ} \mathrm{C}\right)$. During these experiments the retentate and permeate sides of the membrane were continuously flushed at atmospheric pressure, the retentate side with a mixture of hydrogen and helium, the permeate side with pure nitrogen. The flux and selectivity were determined by measuring the hydrogen and helium concentration in the nitrogen stream with a gas chromatograph, equipped with a Thermal Conductivity Detector (TCD).

The hydrogen flow rate through the membrane versus a duration of the experiment is given in figure 8 . It should be noted that the measurement started when the membrane temperature was at $350^{\circ} \mathrm{C}$. At an average membrane

Figure 5: Flow channels etch in glass by the powder blasting

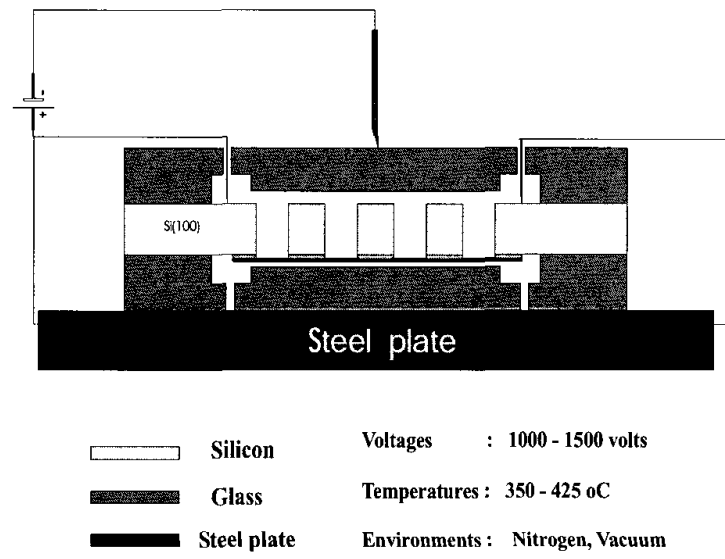

Figure 6: Four electrode anodic bonding setup for double side bonding

temperature of $450^{\circ} \mathrm{C}$, hydrogen flux of $0.5 \mathrm{~mol}$ $\mathrm{H}_{2} / \mathrm{m}^{2}$.s was achieved.

Moreover, dependence of hydrogen flux on temperature was also investigated. Figure 9 shows the hydrogen flow rates through the membrane at different temperatures from $350^{\circ} \mathrm{C}$ to $450^{\circ} \mathrm{C}$. As can be seen that hydrogen flow rate increases with increasing temperature. However, the dependence is stronger than expected by theory [11]. More experiments are needed to be done to have better understanding of the membrane characterizations.

From the measured data, a minimal separation factor of 550 for hydrogen to nitrogen was calculated. The final determination of selectivity was limited by the sensitivity of our equipments.

\section{CONCLUSIONS}

MEMS based wafer-scale palladium - silver alloy membranes were fabricated and tested. Anodic bonding of thick glass to silicon was used to package the membrane and create the very robust module. 


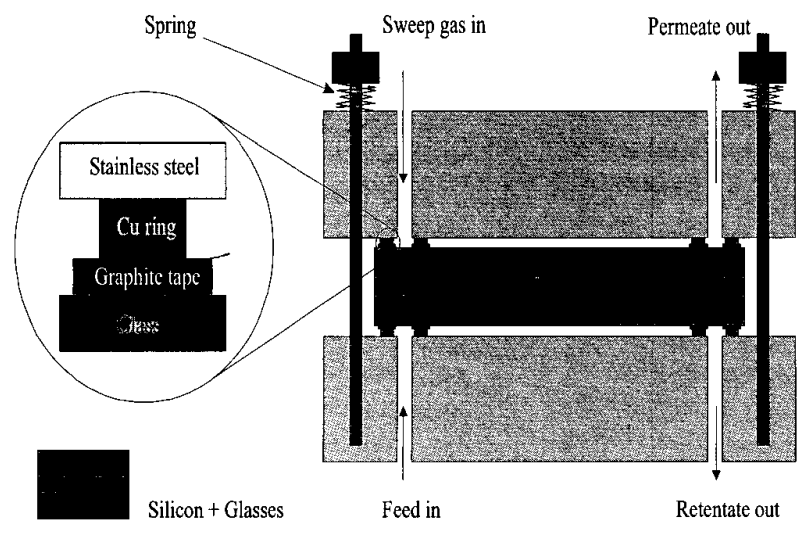

Figure 7: Membrane holder, the upper part can move up and down and is pressed to the lower part by springs.

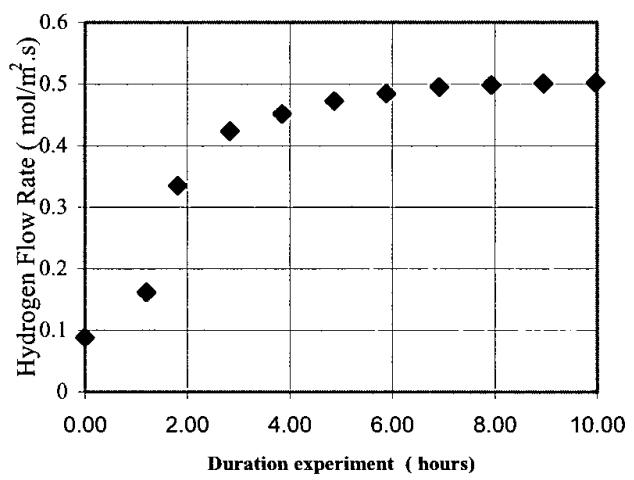

Figure 8: Hydrogen flow rate through the membrane as a function of time at temperature of $450^{\circ} \mathrm{C}$. The measurement started, when the membrane temperature was at $350^{\circ} \mathrm{C}$ in hydrogen environment.

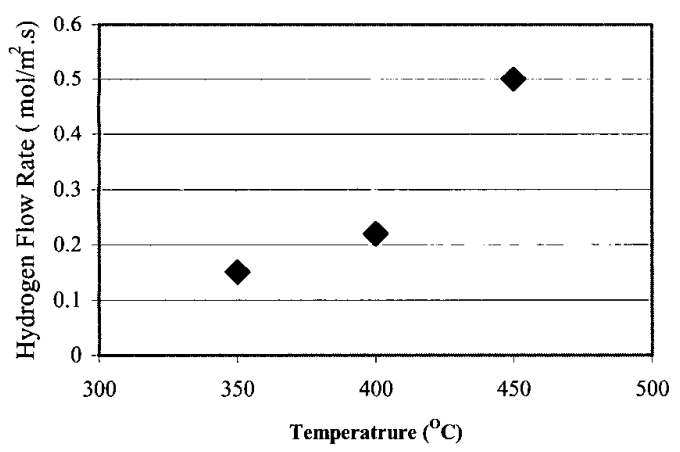

Figure 9: Dependence of hydrogen flow rate through the microfabricated PdAg membrane on temperature.
The membranes have high mechanical strength and are capable of withstanding pressure difference of 4 bars at room temperature. The palladium-silver membranes achieve high hydrogen selectivity and high permeation rate. Typical flow rates of $0.5 \mathrm{~mol}$ $\mathrm{H}_{2} / \mathrm{m}^{2}$.s are measured with the minimal selectivity of 550 for $\mathrm{H}_{2} / \mathrm{N}_{2}$. The reported palladium-silver alloy membranes can be used for hydrogen separation or purification from gas mixture or other industrial applications $[1,2,3]$.

The results indicate that an industrial module that consists of a stack of a number of glass/membrane plates with a higher throughput of hydrogen becomes feasible.

\section{ACKNOWLEDGEMENTS}

The STW foundation is kindly acknowledged for financial support. Our thanks are also due to the entire staff of $\mathrm{MESA}^{+}$, University of Twente, for technical support. H.Wensink graciously provided help with the powder blasting.

\section{REFERENCES}

[1] R.Dittmeyer,V.Hollein and K.Daub, J.Molecular Catalysis A: Chemical 173, 2001, pp.135-184.

[2] R.Hughes, Memb.Tech. 131,2001,pp.9-13.

[3] J.Shu,B.P.A.Grandjean,A.VanNeste, J.Kaliaguine,Can.J.Chem.Eng. 69, 1991, p.1036.

[4] Aleksander J.Frank, Klavs F.Jensen, Martin A.Schmidt,Proc.IEEE Conf. MEMS'99, pp.382387

[5] M.Elwenspoek and H.V.Jansen, Silicon Micromachining, Oxford Press, 1999.

[6] M.Vangbo,Y.Backlund, J.Micromech.Microeng. 6, 1996, pp. 279-284.

[7] J.L.Vossen, W.Kern, Thin Film Processing II, Academic Press, INC., 1991.

[8] Z.Y.Li, J.membr.Sci.78, 1993,pp.247-254.

[9] H.Wensink, M.Elwenspoek, H.V.Jansen, and E.Berenschot, Proc. IEEE Conf. MEMS'00, pp.769-774.

[10] Goodfellow data,Catalogue, 1991/1992.

[11] T.L.Ward,T.Dao, J.Mem.Sci.153,1999, pp.211-231. 